\title{
Induction of MCP1, CCR2, and iNOS Expression in THP-1 Macrophages by Serum of Children Late After Kawasaki Disease
}

\author{
YIU-FAI CHEUNG, KARMIN O, SIDNEY C. F. TAM, AND YAW L. SIOW \\ Department of Paediatrics and Adolescent Medicine [Y.-F.C.], Grantham Hospital, The University of \\ Hong Kong, Hong Kong, China; Departments of Animal Science and Physiology [K.O.], St. Boniface \\ Hospital Research Centre, University of Manitoba, Winnipeg, Manitoba R2H 2A6, Canada; Department of \\ Physiology [K.O., Y.L.S.], Centre for Research and Treatment of Atherosclerosis, University of Manitoba, \\ Winnipeg, Manitoba R3E OW3, Canada; Division of Clinical Biochemistry [S.C.F.T.], Queen Mary \\ Hospital, Hong Kong, China
}

\begin{abstract}
Evidence of premature atherosclerosis late after Kawasaki disease $(\mathrm{KD})$ is accumulating. Given the potential roles of monocyte chemoattractant protein-1 (MCP-1), chemokine receptor CCR-2, and inducible nitric oxide synthase (iNOS) in atherogenesis, we sought to determine whether serum obtained from children late after KD would induce expression of these genes in macrophages in vitro. A total of 79 subjects were studied, which comprised $57 \mathrm{KD}$ patients, 33 of whom had coronary aneurysms, and 22 age-matched controls. Expression of MCP-1, CCR2, and iNOS mRNA in THP-1 macrophages in the presence of patient and control serum was quantified as a ratio to $\beta$-actin mRNA and expressed as a percentage of control. MCP-1 expression was significantly increased in the presence of serum from patients with coronary aneurysms. Expression of CCR2 and iNOS was significantly increased when THP-1 macrophages were incubated with serum from patients with and without coronary an-
\end{abstract}

\section{ABSTRACT}

eurysms. The magnitude of induction of MCP-1, CCR2, and iNOS or in combinations correlated positively with serum highsensitivity C-reactive protein (hs-CRP), and low-density lipoprotein (LDL) cholesterol levels and negatively with high-density lipoprotein (HDL) cholesterol level. In conclusion, the serum of patients with a history of KD induces expression of MCP-1, CCR2, and iNOS in THP-1 macrophages in vitro. Induction of these genes in vivo may be related to chronic inflammation and may have important implications for premature atherosclerosis. (Pediatr Res 58: 1306-1310, 2005)
Abbreviations
hs-CRP, high-sensitivity C-reactive protein
iNOS, inducible nitric oxide synthase
KD, Kawasaki disease
MCP-1, monocyte chemoattractant protein-1

$\mathrm{KD}$ is at present the most common acquired heart disease in children in developed countries (1). It has been more than $25 \mathrm{y}$ since its first description and concerns have been raised regarding the possibility of its predisposition to premature atherosclerosis later in life (2-6). We have previously reported on the adverse cardiovascular risk profile, as characterized by a proatherogenic alteration of lipid profile and increased peripheral conduit arterial stiffness, in children with a history of KD (7). Furthermore, carotid intima-media thickness, a marker of

Received February 28, 2005; accepted April 29, 2005

Correspondence: Y.-F. Cheung, M.D., Division of Paediatric Cardiology, Department of Paediatrics and Adolescent Medicine, Grantham Hospital, The University of Hong Kong, 125 Wong Chuk Hang Road, Hong Kong, China; e-mail: xfcheung @hkucc.hku.hk

Supported in part by the CRCG Research Grant, Faculty of Medicine, The University of Hong Kong.

DOI: 10.1203/01.pdr.0000183360.79872.1c atherosclerosis (8), has been found to be significantly increased in patients studied at about $10 \mathrm{y}$ after the acute illness (9). While there is growing evidence to suggest premature atherosclerosis in patients with a history of $\mathrm{KD}$, the underlying mechanism remains unknown.

Inflammatory processes play a pivotal role in atherogenesis (10). Recently, we (11) and the others (12) have provided evidence that low-grade chronic inflammation may continue unabated after the acute phase of KD. One of the important features of atherosclerosis is infiltration of monocytes into the injured arterial wall followed by their differentiation into macrophages (13). Recent evidence suggests that the inflammatory response in vascular injury involves recruitment and activation of monocytes through activation of the MCP-1 (10,14,15), a potent chemotactic factor for monocytes. In both human and experimental animal models (16), expression of the MCP-1 mRNA and protein has been found to be markedly elevated in atherosclerotic lesions. MCP-1 exerts its action mainly through 
the interaction with the chemokine receptor CCR2 on the surface of monocytes (17).

Apart from the MCP-1/CCR2 pathway, the possible role of iNOS in atherogenesis has also been implicated (18). The iNOS is expressed mainly in the macrophages. Prolonged production of nitric oxide at high levels upon iNOS induction may lead to cell injury in the vessels and may play a role in the atherosclerotic process via mechanisms relating to enhancement of oxidative stress $(19,20)$. Indeed, iNOS expression has been demonstrated in atheromatous plaque (21) and its expression correlates with atherosclerotic intimal thickening (22).

Given the growing evidence of premature atherosclerosis in patients with a history of $\mathrm{KD}$ and the potential roles of MCP-1, CCR2, and iNOS in atherogenesis, we determined in the present study whether the serum obtained from children late after KD would induce the expression of these genes in the macrophages in vitro. We further determined the effects of serum hs-CRP and lipids, known to be altered in the long term after $\operatorname{KD}(7,11,12,23)$ and that may affect chemokine and chemokine receptor expression (24-27), on the magnitude of gene expression.

\section{METHODS}

Subjects. Patients with a history of KD were recruited from the pediatric cardiac clinic of Grantham Hospital. Patients diagnosed to have KD within 12 mo of study were excluded to minimize potential confounding influence relating to subacute inflammation (28). From the medical records, the following data were collected: interval from disease onset to the time of study, coronary complications, and cardiac symptoms at the time of study. Coronary aneurysms were documented by serial two-dimensional echocardiography. Healthy age-matched subjects were recruited as controls. These were healthy children previously discharged from our clinic with a diagnosis of a functional heart murmur and their healthy siblings. The subjects presented for study after an overnight fast. Body weight and height were measured, and body mass index was calculated accordingly. The Institutional Review Board of the University of Hong Kong/Hospital Authority Hong Kong West Cluster approved the study, and parents of all subjects gave written, informed consent.

Blood investigations. Venous blood was withdrawn for measurement of fasting total cholesterol, HDL and LDL cholesterol and triglyceride levels. Serum total cholesterol level was determined enzymatically using a Hitachi 912 analyzer (Roche Diagnostics, GmbH, Mannheim, Germany). HDL cholesterol was measured using a homogeneous method with polyethylene glycolmodified enzymes and sulfated $\alpha$-cyclodextrin. LDL cholesterol was calculated by the Friedewald equation. The serum was separated by centrifugation and stored at $-70^{\circ} \mathrm{C}$ until use. The hs-CRP was measured using a highly sensitive assay, which is a particle-enhanced immunoturbidimetric assay using anti-CRP MAb coupled to latex microparticles (Roche Diagnostics, GmbH). The assay is standardized against CRM 470 Reference Preparation for Proteins in Human Serum (RPPHS) and has a functional sensitivity of $0.1 \mathrm{mg} / \mathrm{L}$.

Cell culture. THP-1 cells, a human monocytic cell line, were purchased from the American Type Culture Collection (Manassas, VA). Cells were cultured in RPMI 1640 medium containing 10\% low endotoxin fetal bovine serum, $100 \mathrm{U} / \mathrm{mL}$ penicillin, and $10 \mu \mathrm{g} / \mathrm{mL}$ streptomycin. For experiments, THP-1 cells were cultured in six-well dishes in the presence of phorbol 12 -myristate 12 -acetate for $16 \mathrm{~h}$ to induce differentiation of these cells into macrophage-like cells (defined as THP-1 macrophages) (29).

Analysis of MCP-1, CCR2, and iNOS mRNA expression. THP-1 macrophages were incubated for $24 \mathrm{~h}$ in RPMI $1640(1 \mathrm{~mL})$ in the presence of serum (10\%, vol/vol). After incubation, total RNA was isolated from cultured cells with TRIzol Reagent (Life Technologies). The MCP-1, CCR2, and iNOS mRNA expressions were examined by semiquantitative reverse-transcriptase (RT-PCR) analysis. The $\beta$-actin was used as an internal standard to verify equal PCR product loading for each experiment. The nucleotide sequences of primers for MCP-1 cDNA were 5'-CAA ACT GAA GCT CGC ACT CTC GCC-3' and 5'-ATT CTT GGG TTC TGG AGT GAG TGT TCA-3'. The nucleotide sequences of primers for $\beta$-actin cDNA were $5^{\prime}$ - GTG GGG CGC CCC AGG CAC CA- $3^{\prime}$ and $5^{\prime}$ - CTC CTT AAT GTC ACG CAC GAT TT- ${ }^{\prime}$. The PCR was performed under the following conditions: initial denaturation for $5 \mathrm{~min}$ at $95^{\circ} \mathrm{C}, 35$ cycles of amplification $\left(95^{\circ} \mathrm{C}\right.$ for $45 \mathrm{~s}, 55^{\circ} \mathrm{C}$ for $50 \mathrm{~s}$, and $72^{\circ} \mathrm{C}$ for $90 \mathrm{~s}$ ), and an additional 10 -min extension at $72^{\circ} \mathrm{C}$. The PCR products were separated by $1.8 \%$ agarose gel (containing $0.5 \mu \mathrm{g} / \mathrm{mL}$ ethidium bromide) electrophoresis. Bands corresponding to MCP-1 mRNA and $\beta$-actin were visualized using autoradiography and analyzed using a gel documentation system (Bio-Rad Gel Doc1000). The ratio of MCP-1 mRNA to $\beta$-actin mRNA was calculated and values were expressed as a percentage of control. Similar experiments were carried out to determine the expression of CCR2 and iNOS mRNA in the THP-1 macrophages. The nucleotide sequences of primers for CCR2 were 5' -ATG CTG TCC ACA TCT CGT TCT CG-3' and 5'-TTA TAA ACC AGC CGA GAC TTC CTG C-3'. The nucleotide sequences of primers for iNOS were 5'-CGG TGC TGT ATT TCC TTA CGA GGC GAA GAA GG-3' and 5'-GGT GCT GCT TGT TAG GAG GTC AAG TAA AGG GC.

Statistical analysis. Data are presented as mean \pm SEM. Differences in demographic data and serum lipid levels between patients and control subjects were compared using unpaired $t$ test. Patients with and without coronary aneurysms were grouped together for comparison with control subjects as they had no statistically significant differences in these variables. For the entire cohort, Pearson correlation analysis was used to assess for possible relationships between gene expression levels and serum hs-CRP, cholesterol, and triglyceride levels. High-sensitivity CRP levels in the patients and controls were expressed as median and interquartile range and compared using MannWhitney $U$ test. As hs-CRP levels showed a skewed distribution, logarithmically transformed CRP values were used in correlation analysis. A $p$ value $<0.05$ was considered statistically significant. All statistical analyses were performed using SPSS version 10.0 (SPSS, Inc., Chicago, IL).

\section{RESULTS}

Subjects. A total of 79 subjects were studied, comprising 57 patients after KD and 22 controls subjects. Of the 57 patients who all had received i.v. immunoglobulin during the acute phase of the illness, 33 had coronary aneurysms. The patients were studied at $7.0 \pm 0.4 \mathrm{y}$ after KD. None were symptomatic and none required coronary arterial interventions. All of the subjects were Chinese in origin. The demographic data of the patients and controls are summarized in Table 1. There were no significant differences in age, sex distribution, and body mass index between patients and controls.

Effects of patient serum on gene expression. The effects of incubating THP-1 macrophages in the presence of serum from patients and controls on MCP-1, CCR2 and iNOS expression are shown in Figure 1.

MCP-1 expression in THP-1 macrophages was significantly greater when incubated with serum from patients with coronary aneurysms $(112.8 \pm 4.1 \%, p=0.015)$ than that for macrophages incubated with control serum (Fig. 1A). On the other hand, incubation with serum from patients without coronary aneurysms did not increase macrophage MCP-1 expression ( $p$ $=0.47$ ).

Table 1. Demographic data and cholesterol levels of patients and controls

\begin{tabular}{lccc}
\hline & $\begin{array}{c}\text { Patients } \\
(n=57)\end{array}$ & $\begin{array}{c}\text { Controls } \\
(n=22)\end{array}$ & $p$ \\
\hline Age $(\mathrm{y})$ & $9.0 \pm 0.4$ & $8.2 \pm 0.4$ & 0.26 \\
Gender $(\mathrm{M} / \mathrm{F})$ & $36 / 21$ & $13 / 9$ & 0.80 \\
BMI $\left(\mathrm{kg} / \mathrm{m}^{2}\right)$ & $16.7 \pm 0.4$ & $16.1 \pm 0.5$ & 0.45 \\
Total cholesterol (mmol/L) & $4.35 \pm 0.10$ & $4.28 \pm 0.15$ & 0.71 \\
HDL cholesterol (mmol/L) & $1.38 \pm 0.03$ & $1.53 \pm 0.07$ & $0.035^{*}$ \\
Total cholesterol/HDL & $3.24 \pm 0.10$ & $2.89 \pm 0.13$ & $0.04^{*}$ \\
$\quad$ cholesterol ratio & & & \\
LDL cholesterol (mmol/L) & $2.61 \pm 0.09$ & $2.33 \pm 0.14$ & 0.10 \\
Triglyceride (mmol/L) & $0.81 \pm 0.04$ & $0.91 \pm 0.08$ & 0.29 \\
\hline
\end{tabular}

* Statistically significant.

BMI, body mass index. 

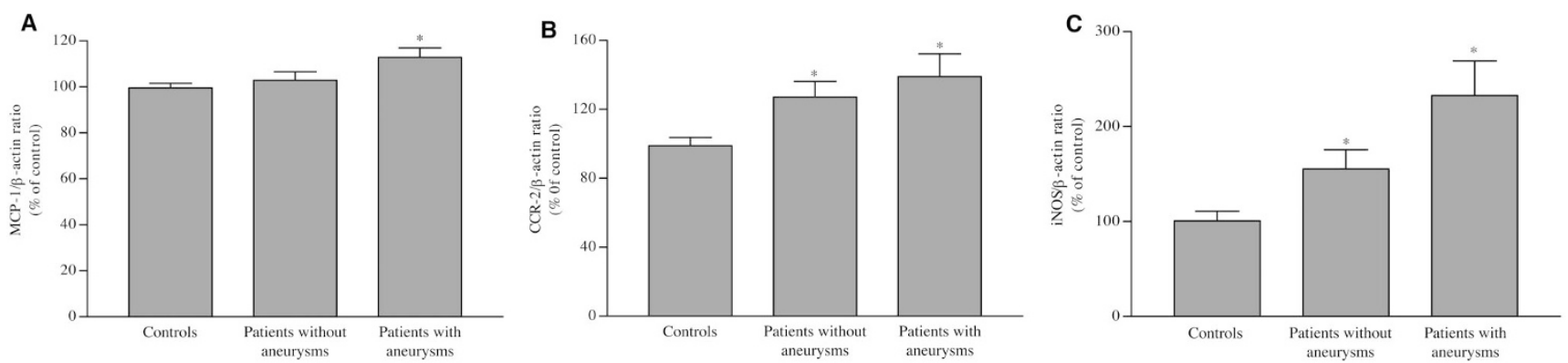

Figure 1. Effect of serum from patients with coronary aneurysm $(n=33)$, patients without coronary aneurysm $(n=24)$, and controls $(n=22)$ on the expression of MCP-1 $(A)$, CCR2 $(B)$, and iNOS $(C)$ in THP-1 macrophages. ${ }^{*} p<0.05$ when compared with control group.

Expression of CCR2 in THP-1 macrophages was significantly greater when incubated with serum both from patients with $(139.0 \pm 13.2 \%, p=0.02)$ and without $(127.2 \pm 9.0 \%$, $p=0.016)$ coronary aneurysms than that for macrophages incubated with control serum (Fig. 1B). Similarly, iNOS expression was significantly greater when incubated with serum both from patients with $(232.5 \pm 36.7 \%, p=0.004)$ and without $(155.3 \pm 20.2 \%, p=0.02)$ coronary aneurysms than that for macrophages incubated with control serum (Fig. 1C).

The possibility of a linear trend of the magnitude of gene expression in relation to the degree of inflammatory vascular damage was further determined using linear regression analysis of gene expression in the three cohorts: controls, patients without coronary aneurysms, and patients with coronary aneurysms. A significant trend was observed for MCP-1 ( $p=$ $0.016)$, CCR2 $(p=0.02)$, and iNOS $(p=0.006)$ expression.

Serum hs-CRP level and gene expression. When compared with healthy controls (median 0.23 , interquartile range 0.17 $0.26 \mathrm{mg} / \mathrm{L}$ ), patients had significantly higher hs-CRP levels (median 0.31, interquartile range $0.22-0.56 \mathrm{mg} / \mathrm{L}, p=0.035$ ). Among patients, those with coronary aneurysms had significantly higher hs-CRP levels (median 0.36, interquartile range $0.27-0.72 \mathrm{mg} / \mathrm{L}$ ) than those without (median 0.25 , interquartile range $0.17-0.40 \mathrm{mg} / \mathrm{L}, p=0.017$ ). Table 2 summarizes the correlations between serum hs-CRP level and MCP-1, CCR2, and iNOS expression in THP-1 macrophages. MCP-1 ( $p=$ $0.031)$ and iNOS $(p=0.034)$ expression correlated positively with hs-CRP level, while CCR-2 expression $(p=0.072)$ also tended to correlate positively with hs-CRP level.

Serum lipids and gene expression. When compared with healthy controls, patients had significantly lower HDL choles-

Table 2. Correlation between serum lipid profile and in vitro expression of MCP-1, CCR-2, and iNOS in THP-1 macrophages

\begin{tabular}{|c|c|c|c|c|c|c|}
\hline & \multicolumn{2}{|c|}{ MCP-1 } & \multicolumn{2}{|c|}{ CCR-2 } & \multicolumn{2}{|c|}{ iNOS } \\
\hline & $r$ & $p$ & $r$ & $p$ & $r$ & $p$ \\
\hline Log hs-CRP (mg/L) & 0.29 & $0.031 *$ & 0.25 & 0.072 & 0.29 & $0.034 *$ \\
\hline Total cholesterol $(\mathrm{mmol} / \mathrm{L})$ & 0.02 & 0.43 & 0.13 & 0.29 & 0.23 & 0.06 \\
\hline HDL cholesterol $(\mathrm{mmol} / \mathrm{L})$ & -0.31 & $0.007^{*}$ & -0.08 & 0.51 & -0.003 & 0.98 \\
\hline $\begin{array}{l}\text { Total cholesterol/HDL } \\
\text { cholesterol ratio }\end{array}$ & 0.28 & $0.016^{*}$ & 0.25 & $0.038 *$ & 0.18 & 0.14 \\
\hline LDL cholesterol $(\mathrm{mmol} / \mathrm{L})$ & 0.25 & $0.038 *$ & 0.27 & $0.026^{*}$ & 0.27 & $0.021 *$ \\
\hline Triglycerides & -0.01 & 0.92 & -0.10 & 0.44 & 0.10 & 0.94 \\
\hline
\end{tabular}

* Statistically significant.

CCR-2, receptor for monocyte chemoattractant protein-1. terol level $(p=0.035)$ and hence a greater total cholesterolto-HDL cholesterol ratio $(p=0.04)$ (Table 1$)$. The LDL cholesterol level also tended to be higher in patients $(p=0.1)$. On the other hand, there were no statistically significant differences in total cholesterol $(p=0.71)$ and triglyceride levels $(p=0.29)$ between patients and controls.

The correlations between serum lipid levels and MCP-1, CCR2, and iNOS expression in THP-1 macrophages are summarized in Table 2. MCP-1 expression correlated positively with LDL cholesterol $(r=0.25, p=0.038)$ and total cholesterol/HDL cholesterol ratio $(r=0.28, p=0.016)$, and negatively with HDL cholesterol $(r=-0.31, p=0.007)$. CCR2 expression similarly correlated positively with LDL cholesterol $(r=0.27, p=0.026)$ and total cholesterol/HDL cholesterol ratio $(r=0.25, p=0.038)$, while iNOS expression correlated positively only with LDL cholesterol $(r=0.27, p=0.021)$.

\section{DISCUSSION}

This study demonstrated significant induction of MCP-1, CCR2, and iNOS expression in THP-1 macrophages in vitro by the serum of children with a history of KD. Furthermore, the serum from patients with coronary aneurysms, compared with that from patients without coronary complications, was found to induce significantly greater expression of these genes. Given the potentially important roles of MCP-1, CCR2, and iNOS in atherogenesis $(10,14-22)$, the findings of the present study provide in vitro evidence that predisposition to premature atherosclerosis in patients after KD may be related to chronic activation of the MCP-1/CCR2 pathway and iNOS in vivo. Additionally, the magnitude of induction was found to correlate with the serum hs-CRP and lipid levels.

Increased MCP-1 expression in the mononuclear cells and increased plasma levels of MCP-1 and monocyte chemotactic activity have been reported in the acute phase of KD (30). Persistent elevation of MCP-1 expression in the peripheral blood mononuclear cells into the subacute and convalescent phases has further been shown in patients with coronary artery lesions (31). Immunohistochemical studies using cardiac tissues of patients with fatal KD revealed that extracellular matrix-bound MCP-1 is associated with mononuclear cellular infiltration and that the sites of MCP-1 expression correlate with the distribution of the acute inflammation, including early coronary vasculitis (32). The findings of the present study, performed at a mean of $7 \mathrm{y}$ after the acute illness, provide 
additional evidence of persistent long-term induction of MCP-1 expression by the serum of patients complicated by coronary aneurysm formation.

MCP-1 exerts its action mainly through the interaction with CCR2, a high-affinity receptor for MCP-1, on the surface of monocytes (17). Unlike MCP-1 gene expression, CCR2 gene expression in the THP-1 macrophages was induced by serum obtained from both patients with and those without coronary complications. Conceivably, upregulation of CCR2 would increase the monocyte responsiveness to MCP-1. To our knowledge, in vitro induction of CCR2 gene expression in macrophages by serum from patients after KD has not been reported previously. In light of the aforementioned findings, it is tempting to speculate that the expression and activity of the MCP1/ CCR2 pathway is chronically increased in vivo in patients even late after resolution of the acute illness KD.

While the cause of MCP-1/CCR2 pathway activation by the $\mathrm{KD}$ serum remains speculative, data in the literature suggest a potential role of chronic inflammation. Sustained expression of MCP-1 has also been reported in chronic inflammation due to rheumatoid arthritis (33). In a rat model of chronic adjuvantinduced vasculitis, increased sensitivity to MCP-1 and increased expression of chemokine receptors CCR 1 and CCR2 in neutrophils have been demonstrated (34). Previous studies showed histologic (35) and biochemical $(11,12)$ evidence of chronic low-grade inflammation persisting for years after the acute phase of KD. Nonetheless, the mechanism by which chronic inflammation activates the MCP-1/CCR pathway requires clarification. It seems, however, unlikely to be related to the proinflammatory cytokines that are elevated during the acute phase as their levels normalize rapidly in the subacute and convalescent phases (36). In fact, interleukin-1, tumor necrosis factor, and interferon gamma decrease, rather than increase, CCR2 gene expression (37). In contrast, CRP has been shown to promote MCP-1-mediated chemotaxis through upregulation of CCR-2 expression in human monocytes (26). Persistent elevation of baseline CRP level after KD $(11,12)$ might be one of the mechanisms that lead to chronic stimulation of the MCP-1/CCR2 pathway.

The associations between MCP-1 and CCR2 expression and serum lipoprotein concentrations are similarly intriguing. We (7) and the others (23) have demonstrated a proatherogenic lipid profile, as characterized by low HDL cholesterol and apolipoprotein A-I levels and a high apolipoprotein B level in $\mathrm{KD}$ patients long after resolution of the acute inflammatory illness. In the present study, the LDL cholesterol levels also tended to be higher in patients than in control subjects. Our findings of positive correlations between CCR2 expression and plasma LDL cholesterol level and total cholesterol/HDL cholesterol ratio agree with those previously reported in patients with hypercholesterolemia (25). The differential effects of plasma lipoproteins on CCR2 expression as illustrated by the reversal of effects of LDL on CCR2 expression by elevated levels of HDL cholesterol has also been reported (25). Animal experiments and in vitro studies have respectively shown that oxidized LDL (38) and enzymatic nonoxidative degradation of LDL (24) lead to induction of MCP-1. The effect of HDL on MCP-1 has not been studied, however. Notwithstanding the dose-dependent correlations between plasma lipoprotein levels and induction of MCP-1/CCR2, the small differences in plasma HDL cholesterol and LDL cholesterol between patients and control subjects suggest that plasma lipoprotein may only play a relatively minor role in modulating the activity of the MCP1/ CCR2 pathway in KD patients.

In human chronic inflammatory diseases, iNOS expression has been localized to the macrophages and epithelial cells among the inflammatory foci (39). Our findings similarly suggest an increase in iNOS expression in macrophages in vivo with inflammation as indicated by its positive correlation with the serum hs-CRP level. In rat macrophages, CRP has been shown to increase iNOS and iNOS mRNA (27). Importantly, the high-output synthesis of nitric oxide is thought to contribute to local tissue destruction in inflammatory conditions involving activated macrophages and neutrophils (40).

The novel findings in the present study of induction of MCP-1/CCR2 pathway and iNOS in macrophages in vitro by the serum of KD patients may have important clinical implications. Increased carotid intima-media thickness (9) and arterial stiffening $(7,9,11)$ have been demonstrated in patients with a history of KD. Correspondingly, the importance of the MCP-1/CCR2 pathway in the pathogenesis of neointimal hyperplasia in cuff-induced vasculitis in mice and monkeys was recently demonstrated (41). Likewise, in a diet-induced atherosclerotic rabbit model, iNOS expression in the aorta has been found to correlate with aortic intimal thickening (22). Induction of these genes in vivo in patients with KD may thus be one of the underlying mechanisms predisposing these patients to premature atherosclerosis. Although the exact pathways by which serum of KD patients induce expression of MCP-1, $\mathrm{CCR}$, and iNOS in macrophages require further clarification, the findings of this study implicate a potential role of chronic low-grade inflammation.

\section{REFERENCES}

1. Taubert KA, Rowley AH, Shulman ST 1994 Seven-year national survey of Kawasaki disease and acute rheumatic fever. Pediatr Infect Dis J 13:704-708

2. Kato H, Sugimura T, Akagi T, Sato N, Hashino K, Maeno Y, Kazue T, Eto G, Yamakawa R 1996 Long-term consequences of Kawasaki disease: a 10- to 21-year follow-up study of 594 patients. Circulation 94:1379-1385

3. Burns JC, Shike H, Gordon JB, Malhotra A, Schoenwetter M, Kawasaki T 1996 Sequelae of Kawasaki disease in adolescents and young adults. J Am Coll Cardiol 28:253-257

4. Kato H, Inoue O, Kawasaki T, Fujiwara H, Watanabe T, Toshima H 1992 Adult coronary artery disease probably due to childhood Kawasaki disease. Lancet 340:1127-1129

5. Ishiwata S, Fuse K, Nishiyama S, Nakanishi S, Watanabe Y, Seki A 1992 Adult coronary artery disease secondary to Kawasaki disease in childhood. Am J Cardiol 69:692-694

6. Silva AA, Maeno Y, Hashmi A, Smallhorn JF, Silverman ED, McCrindle BW 2001 Cardiovascular risk factors after Kawasaki disease: a case-control study. J Pediatr 138:400-405

7. Cheung YF, Yung TC, Tam SC, Ho MH, Chau AK 2004 Novel and traditional cardiovascular risk factors in children after Kawasaki disease:implications for premature atherosclerosis. J Am Coll Cardiol 43:120-124

8. Craven TE, Ryu JE, Espeland MA, Kahl FR, McKinney WM, Toole JF, McMahan MR, Thompson CJ, Heiss G, Crouse JR 3rd 1990 Evaluation of the associations between carotid artery atherosclerosis and coronary artery stenosis. Circulation $82: 1230-1242$

9. Noto N, Okada T, Yamasuge M, Taniguchi K, Karasawa K, Ayusawa M, Sumitomo N, Harada K 2001 Noninvasive assessment of the early progression of atherosclerosis in adolescents with Kawasaki disease and coronary artery lesions. Pediatrics 107:1095-1099

10. Ross R 1999 Atherosclerosis—an inflammatory disease. N Engl J Med 340:115-126

11. Cheung YF, Ho MH, Tam SC, Yung TC 2004 Increased high sensitivity C reactive protein concentrations and increased arterial stiffness in children with a history of Kawasaki disease. Heart 90:1281-1285 
12. Mitani Y, Sawada H, Hayakawa H, Aoki K, Ohashi H, Matsumura M, Kuroe K, Shimpo H, Nakano M, Komada Y 2005 Elevated levels of high-sensitivity C-reactive protein and serum amyloid-A late after Kawasaki disease: association between inflammation and late coronary sequelae in Kawasaki disease. Circulation 111:38-43

13. Ross R 1993 The pathogenesis of atherosclerosis: a perspective for the 1990s. Nature 362:801-809

14. Glass CK, Witztum JL 2001 Atherosclerosis: the road ahead. Cell 104:503-516

15. Lusis AJ 2000 Atherosclerosis. Nature 407:233-241

16. Yla-Herttuala S, Lipton BA, Rosenfeld ME, Sarkioja T, Yoshimura T, Leonard EJ, Witztum JL, Steinberg D 1991 Expression of monocyte chemoattractant protein 1 in macrophage-rich areas of human and rabbit atherosclerotic lesions. Proc Natl Acad Sci U S A 88:5252-5256

17. Charo IF, Myers SJ, Herman A, Franci C, Connolly AJ, Coughlin SR 1994 Molecula cloning and functional expression of two monocyte chemoattractant protein 1 receptors reveals alternative splicing of the carboxyl-terminal tails. Proc Natl Acad Sci U S A 91:2752-2756

18. Sobey CG, Brooks 2nd RM, Heistad DD 1995 Evidence that expression of inducible nitric oxide synthase in response to endotoxin is augmented in atherosclerotic rabbits. Circ Res 77:536-543

19. White CR, Brock TA, Chang LY, Crapo J, Briscoe P, Ku D, Bradley WA, Gianturco $\mathrm{SH}$, Gore J, Freeman BA, et al 1994 Superoxide and peroxynitrite in atherosclerosis. Proc Natl Acad Sci U S A 91:1044-1048

20. Boger RH, Bode-Boger SM, Frolich JC 1996 The L-arginine-nitric oxide pathway: role in the atherosclerosis and therapeutic implications. Atherosclerosis 127:1-11

21. Esaki T, Hayashi T, Muto E, Yamada K, Kuzuya M, Iguchi A 1997 Expression of inducible nitric oxide synthase in T lymphocytes and macrophages of cholesterol-fed rabbits. Atherosclerosis 128:39-46

22. Alfon J, Guasch JF, Berrozpe M, Badimon L 1999 Nitric oxide synthase II (NOS II) gene expression correlates with atherosclerotic intimal thickening. Preventive effects of HMG-CoA reductase inhibitors. Atherosclerosis 145:325-331

23. Newburger JW, Burns JC, Beiser AS, Loscalzo J 1991 Altered lipid profile after Kawasaki syndrome. Circulation 84:625-631

24. Klouche M, Gottschling S, Gerl V, Hell W, Husmann M, Dorweiler B, Messner M, Bhakdi S 1998 Atherogenic properties of enzymatically degraded LDL. selective induction of MCP-1 and cytotoxic effects on human macrophages. Arterioscler Thromb Vasc Biol 18:1376-1385

25. Han KH, Han KO, Green SR, Quehenberger O 1999 Expression of the monocyte chemoattractant protein-1 receptor CCR2 is increased in hypercholesterolemia. Differential effects of plasma lipoproteins on monocyte function. J Lipid Res 40:10531063

26. Han KH, Hong KH, Park JH, Ko J, Kang DH, Choi KJ, Hong MK, Park SW, Park SJ 2004 C-reactive protein promotes monocyte chemoattractant protein-1-mediated chemotaxis through upregulating $\mathrm{CC}$ chemokine receptor 2 expression in human monocytes. Circulation 109:2566-2571
27. Ratnam S, Mookerjea S 1998 The regulation of superoxide generation and nitric oxide synthesis by C-reactive protein. Immunology 94:560-568

28. Fujiwara H, Hamashima Y 1978 Pathology of the heart in Kawasaki disease. Pediatrics 61:100-107

29. Kurosaka K, Watanabe N, Kobayashi Y 1998 Production of proinflammatory cytokines by phorbol myristate acetate-treated THP-1 cells and monocyte-derived macrophages after phagocytosis of apoptotic CTLL-2 cells. J Immunol 161:6245-6249

30. Asano T, Ogawa S 2000 Expression of monocyte chemoattractant protein-1 in Kawasaki disease: the anti-inflammatory effect of gamma globulin therapy. Scand J Immunol 51:98-103

31. Wong M, Silverman ED, Fish EN 1997 Evidence for RANTES, monocyte chemotactic protein-1, and macrophage inflammatory protein- $1 \beta$ expression in Kawasak disease. J Rheumatol 24:1179-1185

32. Terai M, Jibiki T, Harada A, Terashima Y, Yasukawa K, Tateno S, Hamada H, Oana S, Niimi H, Matsushima K 1999 Dramatic decrease of circulating levels of monocyte chemoattractant protein-1 in Kawasaki disease after gamma globulin treatment. J Leukoc Biol 65:566-572

33. Robinson E, Keystone EC, Schall TJ, Gillett N, Fish EN. 1995 Chemokine expression in rheumatoid arthritis (RA): evidence of RANTES and macrophage inflammatory protein (MIP)-1 beta production by synovial T cells. Clin Exp Immunol 101:398-407

34. Johnston B, Burns AR, Suematsu M, Issekutz TB, Woodman RC, Kubes P 1999 Chronic inflammation upregulates chemokine receptors and induces neutrophil migration to monocyte chemoattractant protein-1. J Clin Invest 103:1269-1276

35. McConnell ME, Hannon DW, Steed RD, Gilliland MG 1998 Fatal obliterative coronary vasculitis in Kawasaki disease. J Pediatr 133:259-261

36. Leung DY 1990 Immunologic aspects of Kawasaki syndrome. J Rheumatol Suppl 24:15-18

37. Tangirala RK, Murao K, Quehenberger O. 1997 Regulation of expression of the human monocyte chemotactic protein-1 receptor (hCCRs) by cytokines. J Biol Chem 272:8050-8056

38. Chen YL, Chang YJ, Jiang MJ 1999 Monocyte chemotactic protein-1 gene and protein expression in atherogenesis of hypercholesterolemic rabbits. Atherosclerosis 143:115-123

39. Kroncke KD, Fehsel K, Kolb-Bachofen V 1998 Inducible nitric oxide synthase in human diseases. Clin Exp Immunol 113:147-156

40. Steiner L, Kroncke K, Fehsel K, Kolb-Bachofen V 1997 Endothelial cells as cytotoxic effector cells: cytokine-activated rat islet endothelial cells lyse syngeneic islet cells via nitric oxide. Diabetologia 40:150-155

41. Egashira K, Zhao Q, Kataoka C, Ohtani K, Usui M, Charo IF, Nishida K, Inoue S, Katoh M, Ichiki T, Takeshita A 2002 Importance of monocyte chemoattractan protein-1 pathway in neointimal hyperplasia after periarterial injury in mice and monkeys. Circ Res 90:1167-1172 